\title{
Efeitos de três programas de treinamento na hidroginástica e no subsequente destreinamento sobre a qualidade de vida de mulheres idosas
}

http://dx.doi.org/10.11606/1807-5509201900040629

\author{
Maríndia Teixeira BECKER* \\ Giane Veiga LIEDTKE* \\ Felipe Barreto SHUCH ${ }^{* * * *}$ \\ Luiz Fernando Martins KRUEL"
}

*Universidade Federal do Rio Grande do Sul, Porto Alegre, RS, Brasil.

**Hospital de Clínicas de Porto Alegre, Porto Alegre, RS, Brasil.

\section{Resumo}

0 envelhecimento está relacionado à mudanças estruturais e funcionais do corpo humano, podendo ocasionar alterações no estado emocional e na qualidade de vida (QV). Exercícios físicos, de diferentes modalidades, podem promover melhoras na QV, no entanto, não está claro o quanto diferentes modalidades podem promover diferentes efeitos. Além disso, ainda não está claro qual o período de duração desses benefícios após o término do treinamento. Sendo assim, o objetivo do presente estudo foi comparar três programas de 12 semanas de treinamento na hidroginástica e o subsequente destreinamento nos períodos de quatro e oito semanas, na QV de mulheres idosas. A amostra foi composta por 50 mulheres idosas, randomizadas em três grupos de treinamento na hidroginástica: Grupo de equilibrio, Grupo de força e Grupo aeróbico. 0 período de destreinamento consistiu em uma sessão semanal de alongamento, de 45 minutos em terra. A OV foi analizada com o WHOQL-Old. Após a análise dos dados, foram encontradas melhoras nos domínios psicológico $(p=0,02)$, habilidade sensorial $(p=0,001)$, morte/morrer $(p=0,001)$, autonomia $(p=0,018)$ e intimidade $(p=0,039)$, bem como na QV geral $(p=0,001)$. Não houve diferença entre os diferentes tipos de treinamento. Os incrementos obtidos com o treinamento foram revertidos após o período de oito semanas. Com isso, conclui-se que a hidroginástica pode promover melhoras na QV geral e em aspectos específicos de mulheres idosas, independente do tipo de treinamento. Para tanto, é necessário que se mantenha a regularidade do exercício, visto que oito semanas de destreinamento foram suficientes para reverter os incrementos obtidos pelo treinamento físico.

Palavras-chave: Envelhecimento; Hidroginástica; Qualidade de Vida

\section{Introdução}

O envelhecimento é um processo natural e inevitável, diretamente relacionado a modificaçôes funcionais e estruturais do corpo humano. Essas alterações podem acarretar prejuízos em valências físicas, como o equilíbrio, a força muscular e a capacidade aeróbica ${ }^{1-3}$. Além disso, aspectos psicológicos como o estado emocional e um aumento nos sintomas depressivos podem ocorrer em função do avanço da idade ${ }^{4}$. Dessa forma, tais fatores podem influenciar diretamente na qualidade de vida $(\mathrm{QV})$ do idoso ${ }^{1,3}$.

De acordo com McAuley e MORRIS ${ }^{4}$, a atividade física está diretamente associada à QV. Ainda, o exercício físico também pode gerar incrementos na funcionalidade do idoso, reduzindo os riscos de quedas ${ }^{1,5,6}$. Uma das modalidades bastante indicadas para os idosos é a hidroginástica, pois tem como vantagens um baixo impacto articular nos membros inferiores e o comportamento reduzido da frequência cardíaca e pressão arterial, em virtude das propriedades físicas da água ${ }^{7}$.

Diversos estudos mostram que o treinamento aquático com idosos pode proporcionar benefícios no equilíbrio corporal, na força muscular, na capacidade funcional e na capacidade aeróbica ${ }^{8-13}$. Evidências também suportam os efeitos positivos 
do exercício aquático nos domínios físico e psicológico da QV com a prática sistemática de hidroginástica ${ }^{1,3,14,15}$.

Nesse sentido, alguns autores vêm propondo novos métodos de treinamento na hidroginástica para idosos, os quais apresentam objetivos específicos, como força ${ }^{13}$, equilíbrio ${ }^{16,17}$ e combinado (força e aeróbico $)^{11,18}$. No entanto, nenhum desses estudos avaliou os efeitos desses métodos de treinamento sobre a QV. Além disso, ao nosso conhecimento, não foram encontrados estudos comparando diferentes métodos de treinamento na hidroginástica sobre a QV do idoso.

Apesar dos benefícios do exercício físico sobre a $\mathrm{QV}$, sabe-se que a sua interrupção pode reverter os incrementos obtidos ${ }^{3,19}$. Entretanto, não há dados suficientes que demonstrem o momento em que ocorre o declínio dos incrementos obtidos no treinamento e se o método utilizado pode influenciar a magnitude dessa redução.

Fica claro a relevância do estudo para a área do envelhecimento, visto os benefícios apresentados pela hidroginástica e a falta de evidência dos métodos de treinamento da mesma sobre a QV da população em questão, variável esta tão importante e relacionada a autonomia e independência do idoso, além do aumento da expectativa de vida. Sendo assim, o objetivo do presente estudo foi comparar três programas de 12 semanas de treinamento na hidroginástica e o subsequente destreinamento nos períodos de quatro e oito semanas, sobre a QV de mulheres idosas.

\section{Método}

\section{Participantes}

A amostra foi composta por 50 mulheres idosas, recrutadas de forma não aleatória e por voluntariedade, através da divulgação pelos meios de comunicação (jornais de grande circulação e do bairro local, cartazes e via e-mail). Inicialmente entraram em contato 156 indivíduos, mantendose para a participação no estudo 66 mulheres selecionadas de acordo com os critérios de inclusão e exclusão, que foram divididas aleatoriamente em três grupos de hidroginástica. Das perdas amostrais, três ocorreram no grupo de treinamento com ênfase no equilíbrio corporal (GTE: $\mathrm{n}=$ 17), seis no grupo de treinamento com ênfase na força muscular (GTF: $n=16$ ) e sete no grupo de treinamento com ênfase na capacidade aeróbia (GTA: $\mathrm{n}=17$ ). Foram incluídas no estudo mulheres com idade entre 60 e 75 anos que não praticassem atividade física regular, há no mínimo seis meses antes do início do estudo.

Os seguintes critérios de exclusão utilizados foram: tabagismo, o uso de medicamentos que afetam o sistema muscular e/ou nervoso, acometimentos no sistema nervoso central e periférico, presença de disfunções vestibulares, musculoesqueléticas e ósteoarticulares, doenças cardiovasculares ou prótese em membros inferiores. Além disso, a assiduidade das participantes foi controlada nos treinamentos e considerada um critério de exclusão da análise. O estudo foi aprovado pelo Comitê de Ética da

Universidade Federal do Rio Grande do Sul (no 295.091). Todas as participantes assinaram o termo de consentimento livre e esclarecido e apresentaram eletrocardiograma de esforço ou atestado médico de liberação antes do início do estudo.

\section{Treinamento}

Os três métodos de treinamento tiveram 12 semanas de duração, periodizados em três mesociclos de quatro semanas, com frequência semanal de duas sessões e duração de 45 minutos. Cada sessão foi dividida em aquecimento, parte principal (treinamento de equilíbrio, força ou aeróbico) e volta à calma. $\mathrm{O}$ mesmo profissional de educação física ministrou os diferentes tipos de treinamento, que foram realizados na Escola de Educação Física (ESEF) da Universidade Federal do Rio Grande do Sul (UFRGS), no período de julho a setembro de 2014 .

O treinamento de equilíbrio foi prescrito através da escala de Borg, que tem variação de 6 a 20 , sendo 6 corresponde a nenhum esforço e 20 correspondente ao máximo esforço ${ }^{20}$. Antes do início do treinamento as idosas foram devidamente familiarizadas com a escala de Borg. Os exercícios foram realizados com intensidade no índice de esforço percebido 13, que corresponde a um pouco intenso. $\mathrm{Na}$ parte principal, com duração de 35 minutos, foram realizados exercícios com enfoque no equilíbrio corporal, os quais tiveram 
como principal característica a desestabilização do centro de massa. A progressão do treinamento foi baseada no nível de dificuldade dos exercícios, que consistiram de grandes amplitudes de movimento, com e sem equipamentos, com deslocamentos e estímulos visuais ${ }^{21}$. Foram trabalhados tanto os movimentos de membros inferiores (flexão e extensão do quadril, flexão e extensão do joelho, adução e abdução do quadril) quanto de membros superiores (flexão e extensão horizontal do ombro, adução e abdução dos ombros, flexão e extensão do cotovelo, flexão e extensão do tronco).

A prescrição do treinamento de força foi realizada com intensidade correspondente ao índice de esforço percebido 19: extremamente intenso da escala de Borg, utilizando a máxima resistência proporcionada pelo meio aquático para os exercícios principais. A aula foi dividida em três blocos de exercícios, com volume de 1 minuto para cada grupo muscular. O primeiro mesociclo teve volume total de 23 minutos: 3 séries de 20 segundos com intervalos de 1 minuto e 20 segundos entre as séries. O segundo mesociclo teve volume total de 28 minutos e 30 segundos: 3 séries de 15 segundos com intervalos de 1 minutos e 30 segundos. $\mathrm{O}$ terceiro mesociclo teve volume total de 38 minutos: 2 vezes 3 séries de 10 segundos. $O$ treinamento de força consistiu de movimentos de flexão e extensão do quadril, flexão e extensão do joelho, adução e abdução do quadril, bem como, flexão e extensão horizontal do ombro, adução e abdução do ombro e flexão e extensão do cotovelo. Além disso, entre cada bloco foi realizado o exercício de flexão e extensão do tronco: 2 séries de 30 segundos com intervalo de 2 minutos entre as séries.

Já o treinamento aeróbico foi prescrito em percentuais da frequência cardíaca no segundo limiar ventilatório $\left(\mathrm{FC}_{\mathrm{LV} 2}\right)$, obtida em teste máximo realizado no meio aquático. A intensidade do treinamento variou de $80 \%$ a $95 \%$ da $\mathrm{FC}_{\mathrm{LV} 2}$ de acordo com cada mesociclo. No primeiro mesociclo os exercícios foram executados com a intensidade prescrita em 80 a $85 \%$ da $\mathrm{FC}_{\mathrm{LV} 2}$, o segundo mesociclo com a intensidade prescrita em 85 a $90 \%$ da $\mathrm{FC}_{\mathrm{LV} 2}$ e o terceiro mesociclo com a intensidade prescrita em 90 a $95 \%$ da $\mathrm{F}_{\mathrm{CLV} 2}$. $\mathrm{O}$ treinamento aeróbico foi realizado de maneira contínua, com exercícios específicos da hidroginástica, onde foram associados membros superiores com membros inferiores. A parte principal totalizou 35 minutos e também foram trabalhados movimentos de flexão e extensão do quadril, flexão e extensão do joelho, adução e abdução do quadril, bem como, flexão e extensão horizontal do ombro, adução e abdução do ombro, flexão e extensão do cotovelo e flexão e extensão do tronco.

\section{Destreinamento}

Para o destreinamento da modalidade praticada (hidroginástica), foi realizada após a intervenção somente uma sessão por semana de alongamento em meio terrestre, com duração de 45 minutos, ministrada pelo mesmo professor que aplicou o treinamento na hidroginástica e no mesmo local (ESEF / UFRGS). O destreinamento ocorreu durante 8 semanas com avaliaçóes ao final da quarta semana (primeiro destreinamento) e da oitava semana (segundo destreinamento) após o treinamento. Além disso, a divisão dos grupos foi mantida. Nessas sessões foram executados exercícios de mobilidade articular (mobilização de cervical e ombros) e alongamento passivo de membros superiores (flexores e extensores horizontais do ombro, flexores e extensores do cotovelo e flexores e extensores do punho), membros inferiores (flexores e extensores do quadril, adutores e abdutores do quadril, flexores e extensores do joelho, flexores plantares) e tronco (flexores da coluna cervical, flexores laterais da coluna cervical e extensores da coluna lombar e torácica), com séries únicas de 30 segundos.

\section{QV}

A QV foi mensurada através dos questionários: The World Health Organization Quality of Life Instrument (WHOQOL-BREF), composto por 26 itens agrupados em quatro domínios (físico, psicológico, meio ambiente, relação social) e QV geral; e The World Health Organization Quality of Life Instrument-old version (WHOQOLOLD), módulo da QV aplicado juntamente com o WHOQOL-BREF, dotado de 24 itens específicos para a terceira idade, sendo constituído de seis domínios (habilidade sensorial, autonomia, atividades passadas/presentes/futuras, participação social, morte/morrer, intimidade) e QV num aspecto geral $^{22,23}$. Os questionários foram respondidos com referência nas duas últimas semanas e os escores variaram de zero (pior QV) a 100 (melhor QV) pontos. Os instrumentos foram aplicados no início e final do treinamento, e nas quatro e oito semanas subsequentes após o treinamento. 


\section{Análise Estatística}

Os resultados foram descritos através de valores de média, desvio e erro padrão. Foi realizado teste de Shapiro-Wilk para normalidade dos dados e teste de Levene para homogeneidade dos dados. Para a análise dos dados realizou-se as Equações
Estimadas Generalizadas (GEE). A análise estatística foi realizada no programa estatístico SPSS vs. 15.0, com índice de significância 0,05 . As diferenças entre os grupos e entre os tempos foram analisadas com o teste de post-hoc de bonferroni. Os resultados da GEE serão exibidos em médias ajustadas pelo modelo.

\section{Resultados}

Os grupos não apresentaram diferenças significativas em nenhuma das variáveis: idade, altura, massa corporal, índice de massa corporal e percentual de gordura na medida de base (TABELA 1).

Não foram encontradas diferenças significativas nos fatores principais tempo e grupo, assim como na interação tempo* grupo na percepção geral da QV e nos domínios físico, meio ambiente e participação social do WHOQOL-BREF.

Todavia, foi encontrada uma interação tempo*grupo significativa $(\mathrm{p}=0,02)$ no domínio psicológico, a qual o post-hoc de Bonferroni mostrou que apenas o GTA apresentou uma melhora significativa do período pré treinamento (Média: 65,55, EP: $\pm 4,22$ ) para o pós treinamento (Média: 76,89, EP: $\pm 2,85$ ), mantendo essa adaptação ao longo das demais avaliaçōes. Além disso, o GTE apresentou um declínio significativo $(\mathrm{p}=0,034)$ do período pós treinamento (Média: 77,60, EP: $\pm 1,76$ ) para o segundo destreinamento (Média: 71,52, EP: \pm 1,52). Já o GTF não apresentou qualquer diferença significativa entre os momentos (FIGURA 1 e 2).

TABELA 1 -Caracterização da amostra: Valores de média e desvio-padrão (DP) da idade, estatura, massa corporal, índice de massa corporal (IMC) e percentual de gordura da amostra.

\begin{tabular}{|c|c|c|c|c|c|c|c|}
\hline \multirow[b]{2}{*}{ Variáveis } & \multicolumn{2}{|c|}{ GTE $(n=17)$} & \multicolumn{2}{|c|}{ GTF $(n=16)$} & \multicolumn{2}{|c|}{ GTA $(n=17)$} & \multirow[b]{2}{*}{$\mathrm{p}$} \\
\hline & Média & $\pm \mathrm{DP}$ & Média & $\pm \mathrm{DP}$ & Média & $\pm \mathrm{DP}$ & \\
\hline Idade (anos) & 66,81 & 4,1 & 64,94 & 4,4 & 64,00 & 4,2 & 0,327 \\
\hline Estatura (m) & 1,56 & 0,04 & 1,58 & 0,04 & 1,57 & 0,05 & 0,701 \\
\hline Massa corporal (kg) & 68,95 & 3,55 & 69,73 & 7,66 & 71,70 & 12,29 & 0,684 \\
\hline $\operatorname{IMC}\left(\mathrm{kg} / \mathrm{m}^{2}\right)$ & 28,48 & 1,38 & 29,14 & 5,13 & 28,79 & 4,97 & 0,914 \\
\hline Percentual de gordura $(\%)$ & 31,72 & 5,40 & 32,80 & 4,72 & 34,64 & 6,26 & 0,861 \\
\hline
\end{tabular}

Já nos resultados do WHOQOL-OLD (FIGURA 3, 4 e 5), não foram observadas diferenças significativas nos domínios de atividades passadas/presentes/ futuras e participação social em todos os momentos da intervenção.

Em contrapartida, apresentaram uma melhora significativa $(\mathrm{p}=0,001)$ no fator tempo a percepção geral da QV do período pré treinamento (Média 58,14, $\mathrm{EP}: \pm 1,39$ ) para o pós treinamento (Média: 72,22 EP: $\pm 1,46$ ) e o domínio habilidade sensorial, também do período pré treinamento (Média: 32,61, EP: $\pm 1,98$ ) para o pós treinamento (Média: 78,74, EP: $\pm 2,84$ ), independente do grupo. Havendo uma manutenção dos resultados no primeiro destreinamento da percepção geral da QV (Média: 72,87, EP $= \pm 2,05$ ) e do domínio habilidade sensorial (Média: 80,98, $\pm 3,86$ ), com retorno aos níveis inicias no segundo destreinamento da percepção geral da QV (Média:
59,76, EP: $\pm 2,72)$ e do domínio habilidade sensorial (Média: 31,34, EP: $\pm 2,88$ ), independente do grupo.

$\mathrm{O}$ domínio morte e morrer também apresentou uma diferença significativa no fator tempo do período pré treinamento (Média: 32,03, EP: $\pm 3,50$ ) para o pós treinamento (Média: 63,35, EP: $\pm 4,16$ ) e para o primeiro destreinamento $(72,70 \pm 4,10)$, com retorno aos níveis iniciais também somente no segundo destreinamento $(25,29 \pm 4,78)$, independente do grupo.

O domínio de intimidade apresentou uma diferença significativa $(\mathrm{p}=0,039)$ no fator tempo, porém o posthoc de Bonferroni não identificou em qual momento ocorreu essa significância. Por fim, o domínio de autonomia apresentou uma interação tempo*grupo significativa $(\mathrm{p}=0,018)$, entretanto, o post-hoc de Bonferroni também não identificou em que momento ocorreu essa interação. 

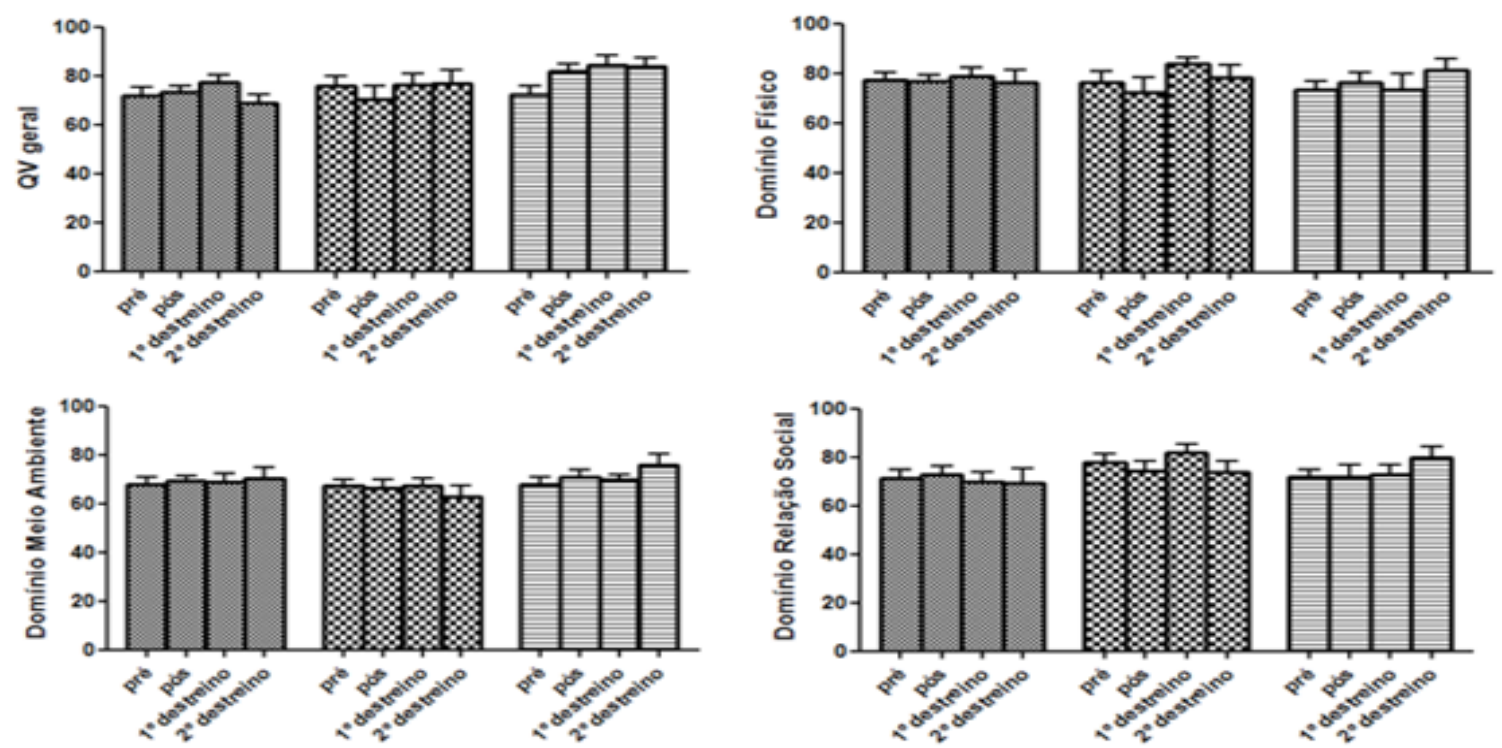

Grupo Equilibrio $\infty$ Grupo Força $\sqsupseteq$ Grupo Aeróbico

FIGURA 1-WHOQOL-BREF.

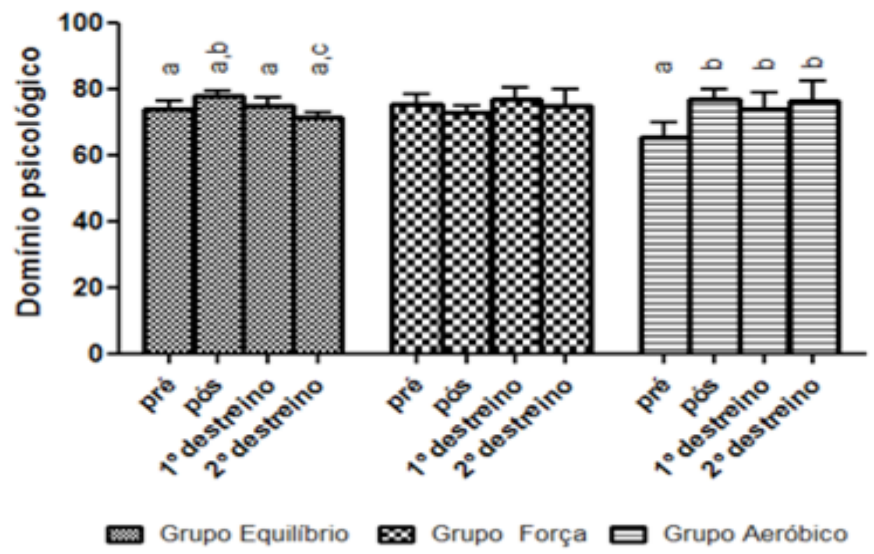

FIGURA 2-WHOQOL-BREF: $\mathrm{p}$ < 0,05. Letras diferentes indicam diferença significativa no mesmo grupo.
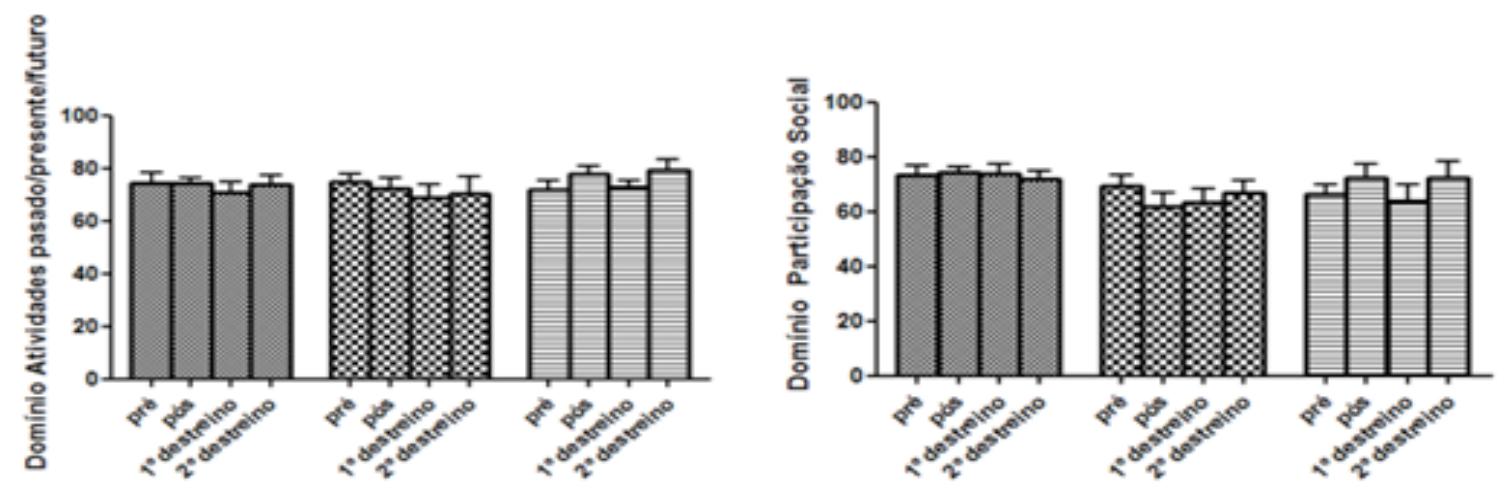

FIGURA 3 - WHOQOL-OLD. 

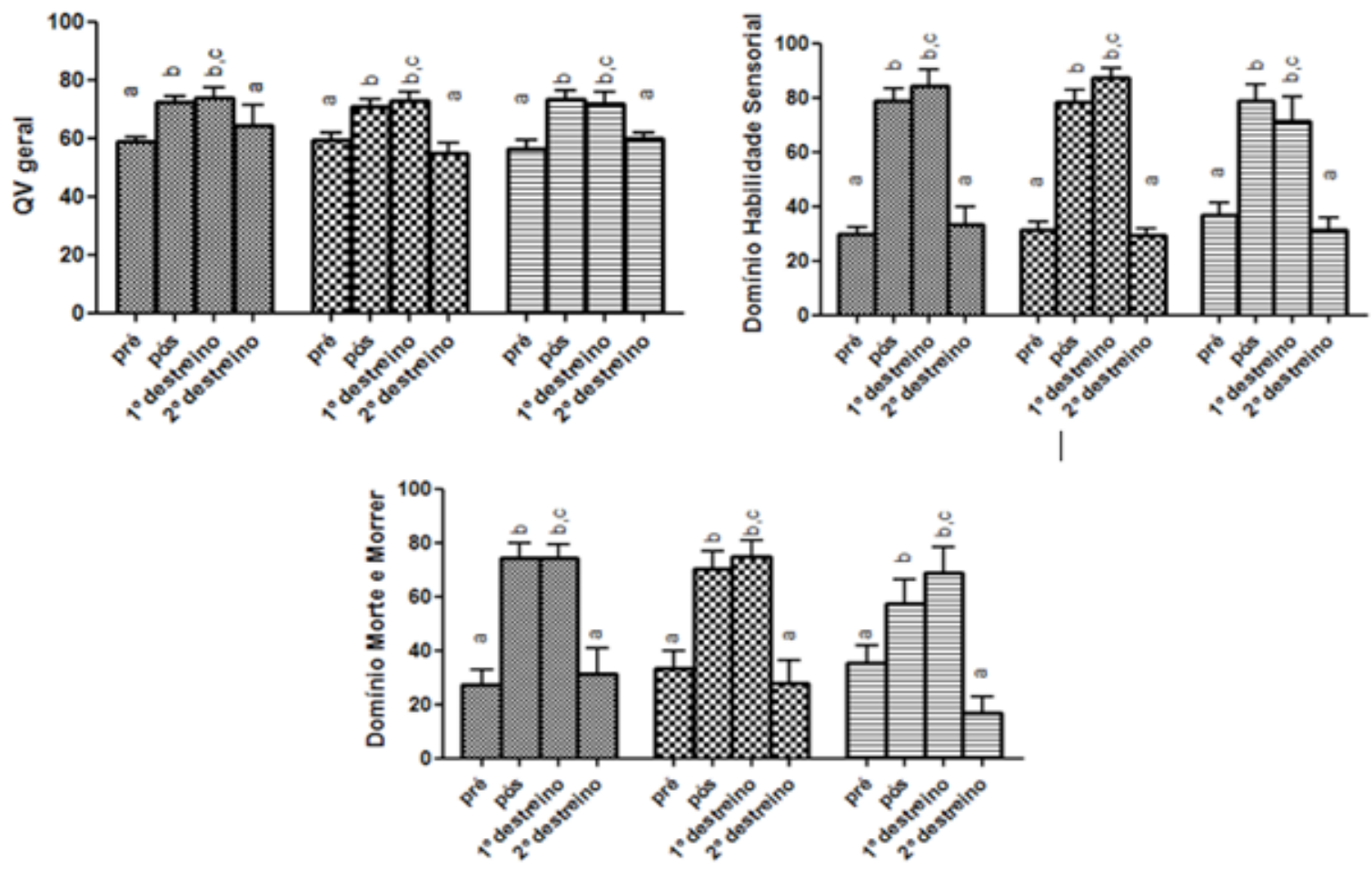

Grupo Equilibrio $\sum \infty$ Grupo Força $\square$ Grupo Aeróbico

FIGURA 4-WHOQOL-OLD: $\mathrm{p}<\mathrm{0,05}$. Letras diferentes indicam diferença significativa no mesmo grupo, no fator tempo.
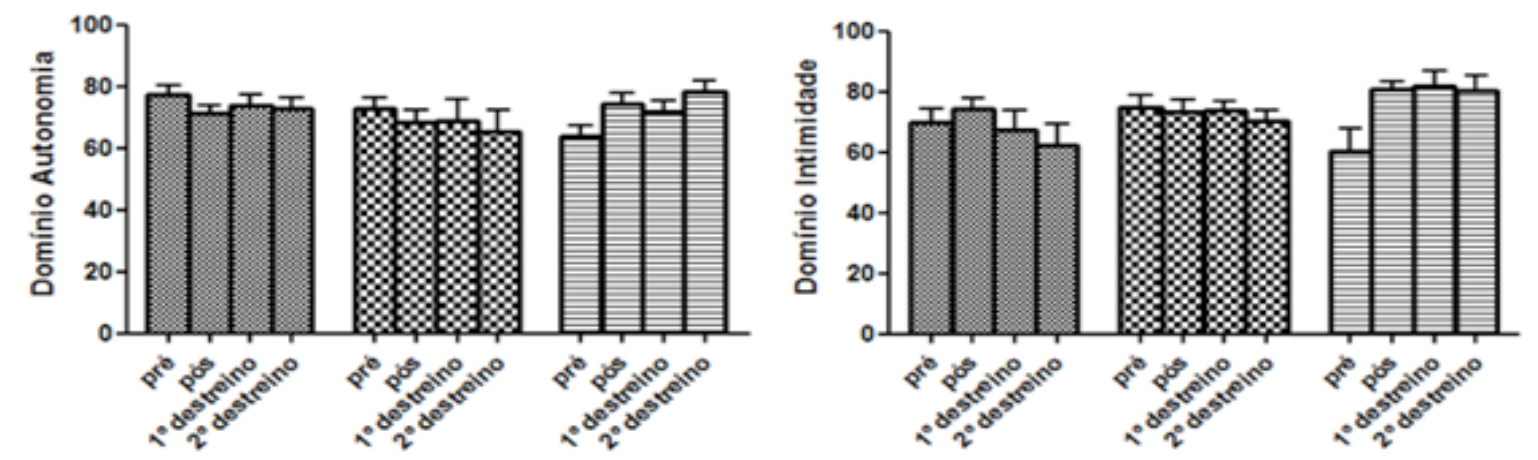

Grupo Equilibrio $\infty$ Grupo Força $\boxminus$ Grupo Aeróbico

FIGURA 5 - WHOQOL-OLD.

\section{Discussão}

Através dos resultados do presente estudo, verificou-se que o treinamento na hidroginástica, independente do método utilizado (GTE, GTF e GTA), foi capaz de promover melhoras na QV geral de mulheres idosas avaliada através do WHOQOL-
OLD. O GTA mostrou uma importante melhora no domínio psicológico e os três grupos nos domínios específicos da terceira idade: habilidade sensorial, morte e morrer e intimidade. A magnitude do efeito entre os grupos variou de fraca a forte, a depender 
do domínio da QV. Ainda, foi possível perceber um declínio significativo nos escores do GTE no segundo destreinamento, mostrando a importância de se manter o exercício físico regularmente.

Devereux, Robertson e Briffa ${ }^{1}$ avaliaram a QV de mulheres idosas após 10 semanas de treinamento na hidroginástica, que teve como ênfase o equilíbrio. Foi realizado o método de treinamento combinado composto por alongamentos, tai chi, caminhada, exercícios aeróbicos e de força, postura, propriocepçáo e equilíbrio. Após a intervenção, observou-se melhoras na QV nos domínios capacidade funcional, vitalidade, aspectos sociais e saúde mental do grupo exercício, quando comparado ao grupo controle. Esses achados vão ao encontro do nosso estudo, que também apresentou melhoras na QV. Entretanto, os estudos diferem em relação ao uso de instrumentos de avaliação da qualidade de vida que possuem bases conceituais diferentes SF-36 x WHOQOL (dotado de oito domínios, que possui uma base conceitual diferente do WHOQOL).

O estudo de TOMAs-Carus et al. ${ }^{19}$ também avaliou a QV de mulheres (idades entre 35 e 73 anos) na hidroginástica, encontrando efeitos positivos após o treinamento. A intervenção proposta consistia de três sessões semanais com duração de $1 \mathrm{~h}$, pelo período de 12 semanas. O método de treinamento realizado foi o combinado composto por exercícios aeróbicos, globais e força. Todavia, além da diferença de idade, não foi mencionado uma progressão do treinamento e o instrumento utilizado também foi o SF-36, limitando as nossas comparaçōes. O estudo ainda avaliou 12 semanas de destreinamento, mantendo o efeito benéfico em apenas dois domínios após esse período.

Corroborando o presente estudo, BoCALINI et al. ${ }^{3}$ verificaram melhora significativa na $\mathrm{QV}$ de idosas após o treinamento na hidroginástica com duração de 12 semanas e três sessôes semanais de 1 h.O método utilizado foi o combinado com exercícios aeróbicos e localizados com equipamentos. O instrumento utilizado para a avaliação da $\mathrm{QV}$ foi o WHOQOL-BREF. Divergindo de nossos achados, nesse estudo verificou-se incrementos significativos em todos os domínios do instrumento, enquanto observamos melhora significativa apenas no domínio psicológico. Esses autores também avaliaram os efeitos da interrupção do treinamento e seus resultados demonstraram uma redução dos ganhos após quatro semanas de destreinamento da modalidade treinada, retornado aos níveis iniciais após seis semanas destreinamento. Esses achados reforçam a importância da manutenção do exercício físico, além de mostrar a eficácia dos métodos de treinamento após um curto período de destreinamento. No presente estudo, os incrementos obtidos com o exercício físico também foram reduzidos após o período de oito semanas de destreinamento, corroborando ainda mais com esses achados.

O exercício físico pode ter um efeito positivo na QV, melhorando a mesma através de vários possíveis mediadores, dentre os quais, melhoras no funcionamento físico (limitaçôes, incapacidades, perfomance e composição corporal), na função cognitiva (atenção e memória) e na função autorelatada (auto-estima, auto-eficácia e afeto), teriam um papel importante ${ }^{4}$. Nessa direção, o efeito positivo na QV, segundo Elavsky et al. ${ }^{24}$, indica que altos níveis de auto-eficácia e afeto positivo estão associados com níveis mais altos de $\mathrm{QV}^{24}$, assim como a diminuição dos sintomas depressivos parece estar diretamente associada a essa melhora da $\mathrm{QV}^{14}$. Tudo isto porque os efeitos da atividade física sobre a QV, mediados por resultados psicológicos, mostram a longo prazo melhoras sobre o bem-estar ${ }^{24}$.

Todavia, a ausência de diferenças significativas entre os três métodos de treinamento pode ser explicada pela falta de especificidade do mesmo, relacionada a parâmetros psicológicos e emocionais. Além disso, uma possível limitação do estudo é o $\mathrm{n}$ amostral, que pode não ter sido suficiente para detectar diferenças nessa população. Outra possível limitação é a falta de um grupo controle. Ainda, até o presente momento, não estão claras quais as características do exercício estáo mais associadas a uma melhora da QV.

Dessa forma, concluímos com o presente estudo que a hidroginástica pode promover melhoras na QV geral e em aspectos específicos de mulheres idosas, independente do tipo de treinamento. Contudo, é extremamente importante que o exercício físico seja mantido regularmente, para que os incrementos obtidos através do treinamento não sejam perdidos. Pois, o estudo em questão mostrou que o período de destreinamento, com alongamento somente uma vez por semana, não foi suficiente para a manutenção da qualidade de vida, tendo os ganhos obtidos com o treinamento de hidroginástica revertidos em oito semanas de destreinamento.

Como aplicação prática nossos achados sugerem importantes resultados para a literatura, demonstrando que os três métodos de treinamento na hidroginástica foram eficazes na melhora da QV 
do idoso. Com isso, além do idoso poder escolher o método de sua preferência, o profissional também pode realizar diferentes métodos para atingir os mesmos resultados relacionados à QV. Além disso, foi possível perceber que apenas oito semanas de destreinamento foram suficientes para a perda dos ganhos obtidos com o exercício físico na QV, mostrando a importância da sua prática sistemática.

\title{
Agradecimentos
}

Os autores agradecem o apoio da Coordenação de Aperfeiçoamento de Pessoal de Nível Superior (CAPES), Conselho Nacional de Desenvolvimento Científico e Tecnológico (CNPq) e Pró-Reitoria de Pesquisa e Pós-Graduação (PROPESQ).

\begin{abstract}
Aging is related to structural and functional changes of the human body and may cause changes in emotional state and quality of life (QOL). Physical exercises of different modalities can promote improvements in $\mathrm{QoL}$, however, it is unclear how much different modalities can promote different effects. Moreover, it is not yet clear what the duration of those benefits after the training. Thus, the aim of this study was to compare three 12-week training programs in water aerobics and subsequent detraining in periods of four and eight weeks, on the QoL of elderly women. The sample consisted of 50 elderly women, randomized into three training groups in aerobics: Balancing Group, strength Group and aerobic group Group. The detraining period consists of a weekly session of stretching, 45 minutes on the land. The $00 \mathrm{~L}$ was analyzed with WHOOL-old. After analyzing the data, improvements were found in the psychological $(p=0,02)$, sensory ability $(p=0,001)$, death/dying $(p=0,001)$, autonomy $(p=0,018)$ and intimacy $(p=0,039)$, as well as in overall $\mathrm{CoL}(p=0,001)$. There were no differences between the different types of training. The increases obtained with the training were reversed after the eight-week period. Thus, it is concluded that the water aerobics can promote improvements in overall $\mathrm{QoL}$ and specific aspects of elderly women, regardless of the type of training. Therefore, it is necessary to maintain the regularity of exercise, since eight weeks of detraining was enough to reverse the increases obtained by physical training.
\end{abstract}

KeYwordS: Aging; Water Aerobics; Quality of Life.

\section{Referências}

1. Devereux K, Robertson D, Briffa NK. Effects of a water-based program on women 65 years and over: a randomised controlled trial. Aust J Physiotherapy. 2005;51:102-108.

2. Rebelatto JR, Castro AP, Sako FK, Aurichio TR. Equilíbrio estático e dinâmico em indivíduos senescentes e o índice de massa corporal. Fisioter Mov. 2008;21(3):69-75.

3. Bocalini DS, Serra AJ, Rica RL, Santos L. Repercussions of training and detraining by water based exercise on functional fitness and quality of life: a short-term follow-up in healthy older women. Clinics. 2010;65(12):1305-1309.

4. McAuley E, Morris K. Advances in Physical Activity and Mental Health: Quality of Life. Am J Lifestyle Med. 2007;1(5):389-396.

5. Sociedade Brasileira de Geriatria e Gerontologia. Queda em Idosos: Prevenção. Rio de Janeiro (RJ): Associação médica brasileira e Conselho federal de medicina; 2008.

6. Sherrington C, Tiedemann A, Fairhall N, Close J, Lord SR. Exercise to prevent falls in older adults: an updated metaanalysis and best practice recommendations. NSW Public Health Bull. 2011;22(3-4):78-83.

7. Kruel LFM. Alteraçôes fisiológicas e biomecânicas em indivíduos praticando exercícios de hidroginástica dentro e fora d'água [tese]. Santa Maria (RS): Universidade Federal de Santa Maria; 2000. 
8. Simmons V, Hansen PD. Effectiveness of water exercise on postural mobility in the well elderly: An experimental study on balance enhancement. J of Gerontology. 1996;51:233-238.

9. Takeshima N, Rogers ME, Watanabe WF, Brechue WF, Okada A, Yamada T, et al. Water-based exercise improves health-related aspects of fitness in older women. Med Sci Sports Exerc. 2002;33(3):544-551.

10. Lord SR, Matters B, George RS, Thomas M, Bindon J, Chan DK, et al. The effects of water exercise on physical functioning in older people. Aust J Ageing. 2006;25(1):36-41.

11. Tsourlou T, Benik A, Dipla K, Zafeiridis A, Kellis S. The effects of a twenty-four-week aquatic training program on muscular strength performance in healthy elderly women. J Strength Cond Res. 2006;20(4):811-818.

12. Avelar NCP, Bastone AC, Alcântara MA, Gomes WF. Efetividade do treinamento de resistência à fadiga dos músculos dos membros inferiores dentro e fora d'água no equilíbrio estático e dinâmico de idosos. Rev Bras Fisioter. 2010;14(3):229236.

13. Graef FI, Pinto RS, Alberton CL, Lima WC, Kruel LFM. The effects of resistance training performed in water on muscle strength in the elderly. J Strength Cond Res. 2010;24:3150-3156.

14. Schuch FB, Pinto SS, Bagatini NC, Zaffari P, Alberton CL, Cadore EL, et al. Water-Based Exercise and Quality of Life in Women: The Role of Depressive Symptoms. Women \& Health. 2014;54:161-175.

15. Schuch FB, Schoenell MCW, Tiggemann CL, Noll M, Alberton CL, Kruel LFM. The effects of water-based strength exercise on quality of life in young women. Sport Sci Health. 2016;12(1):105-112.

16. Abbasi A, Sadeghi H, Tabrizi HB, Bagheri K, Ghasemizad A, Karimi A. Effect of whole body vibration, aquatic balance and combined training on neuromuscular performance, balance and walking ability in male elderly able-bodied individual. World Appl Sci J. 2011;15(1):84-91.

17. Elbar O, Tzedek I, Vered E, Shvarth G, Friger M, Melzer I. A water-based training program that includes perturbation exercises improves speed of voluntary stepping in older adults: A randomized controlled cross-over trial. Arch Gerontol Geriatr. 2013;56(1):134-40.

18. Alves RV, Mota J, Costa MC, Alves JGB. Aptidão física relacionada à saúde de idosos: influência da hidroginástica. Rev Bras Med Esporte. 2004;10(1):31-37.

19. Tomas-Carus P, Häkkinen A, Gusi N, Leal A, Häkkinen K, Ortega-Alonso A. Aquatic Training and Detraining on Fitness and Quality of Life in Fibromyalgia. Med Sci Sports Exerc. 2007;39(7):1044-50.

20. Borg G. Psychophysical bases of perceived exertion. Med Sci Sports Exerc. 1982;14(5):377-381.

21. Melzer I, Elbar O, Tsedek I, Oddsson LI. A water-based training program that include perturbation exercises to improve stepping responses in older adults: study protocol for a randomized controlled cross-over trial. BMC Geriatr. 2008;8:19.

22. Fleck MPA, Louzada S, Xavier M, Chachamovich E, Vieira G, Santos L, et al. Aplicação da versão em português do instrumento abreviado de avaliação da qualidade de vida WHOQOL-bref. Rev Saúde Públ. 2000;34(2):178-183.

23. Fleck MPA, Chachamovich E, Trentini C. Development and validation of the Portuguese version of the WHOQOLOLD module. Rev Saúde Públ. 2006;40(5):785-91.

24. Elavsky S, McAuley E, Robert WM, James FK, Marquez DX, Hu L, et al. Physical Activity Enhances Long-Term Quality of Life in Older Adults: Efficacy, Esteem, and Affective Influences. Ann Behav Med. 2005;30(2):138-145.

ENDEREÇO DE CORRESPONDÊNCIA: Maríndia Teixeira Becker

Rua Felizardo, 750 - Jardim Botânico Porto Alegre - RS - BRASIL

CEP: 90690-200

E-mail: marindia.becker@gmail.com
Submetido: 19/07/2016

Revisado: 16/11/2016

Aceito: 18/05/2017 